\title{
EFECTO DE LA RENTABILIDAD FINANCIERA Y OPERATIVA EN EL PRECIO DE LAS ACCIONES DE LAS EMPRESAS INDUSTRIALES
}

\author{
EFFECT OF FINANCIAL AND OPERATING PROFIT IN THE PRICE OF \\ THE SHARES OF INDUSTRIAL COMPANIES
}

Elisabeth Teresa Casamayou Calderón Universidad Nacional Jorge Basadre Grohmann Tacna, Perú

ORCID: http://orcid.org/0000-0003-3481-3141 Correo electrónico: elisabeth@casamayou.info

\section{RESUMEN}

Objetivo: El estudio de investigación tiene como objetivo determinar el efecto simultáneo e independiente de la rentabilidad financiera y operativa sobre el precio de la acción (PA) de las empresas en estudio. Método: El diseño de estudio es no experimental, con enfoque cuantitativo. El nivel de la investigación es transversal por ser datos de un periodo de 10 años y de tipo correlacional porque sirvió para establecer relación entre las variables. Se tomaron en cuenta las 34 empresas del sector industrial que cotizan en la Bolsa de Valores de Lima. Para la tabulación de los datos se realizó el análisis de correlación, posteriormente el modelo de regresión simple (ROE con PA y ROA con PA) y regresión múltiple (ROE y ROA con PA), para la comprobación de las hipótesis se realizó el análisis de Anova para observar si influyen o no positivamente, finalmente se realizó el análisis de coeficientes para determinar los valores de la ecuación de la recta. Resultados: Los resultados obtenidos son los siguientes: El ROE y el ROA de manera independiente influyen positivamente y explican el comportamiento del precio de la acción en un 27,14 \% y 26,03 \%. En el análisis simultáneo del ROE y ROA sobre el precio de la acción también influyen positivamente en el PA, y explican un $21,80 \%$ del comportamiento del precio de la acción. Conclusiones: Se concluye que el comportamiento del precio de la acción no es un referente de la rentabilidad financiera (ROE), ni la rentabilidad operativa (ROA) de las empresas del sector industrial analizadas, debido a que su impacto no es significante, pero si positivo.

Palabras clave: Rentabilidad; precio de la acción; ratios; Bolsa de Valores; inversionistas; cotización.

\begin{abstract}
Objective: The study of the research has as objective to determine the simultaneous and independent effect of the financial and operating profitability on the share price (SP) of the companies under study. Method: The design of study is Not experimental with a quantitative approach, the level of the investigation is transversal because it is data has a period of 10 years and correlational because it worth to establish a relationship between the variables. The 34 companies of the industrial sector that are listed on the Lima Stock Exchange were taken into account. For the tabulation of the data, the correlation analysis was carried out after the simple regression model (ROE with PA and ROA with SP) and multiple regression (ROE, ROA with SP), to check the hypotheses the Anova Analysis Anova was used to observe if they influence or not positively, finally the analysis of coefficients was performed to determine the values of the equation of the line. Results: The results obtained are the following: ROE and ROA independently influence positively and explain the behavior of the share price by $27.14 \%$ and $26.03 \%$. In the simultaneous analysis of the ROE and ROA on the share price they also positively influence the AP, and explain $21.80 \%$ of the behavior of the share price.Conclusions: It is concluded that the behavior of the share price is not a reference of the financial profitability $(R O E)$ nor the operational profitability $(R O A)$ of the companies of the industrial sector analyzed, due to their impact is not significant but positive.
\end{abstract}

Keywords: Profitability; share price; ratios; Stock Exchange; Investors; quotation. 


\section{INTRODUCCIÓN}

Actualmente, se tiene conocimiento que los inversionistas de las empresas bursátiles están en la búsqueda de nuevas oportunidades para invertir, es por ese motivo, que indicadores bursátiles como el precio de la acción es el reflejo del estado actual de la empresa que evidencia su rentabilidad financiera (rentabilidad sobre recursos propios) o rentabilidad económica (rentabilidad sobre los activos).

De acuerdo a Barakat (2014): "Analizar el impacto de la estructura financiera sobre el valor de las acciones permite que el analista financiero pueda predecir el valor futuro de la compañía” (p.55).

Pražák y Stavárek (2017) afirman lo siguiente: "Los precios de las acciones cambian en respuesta al comportamiento de una serie de variables. Algunas de estas variables importantes son: ganancias, dividendos, proyecciones de flujo de efectivo, activos netos, rendimiento del capital empleado, relación deuda / capital, etc" (p.2).

Lee y Zhang (2012) señalan lo siguiente: "Los hallazgos muestran que la productividad de una empresa, medida por su rendimiento sobre el capital (ROE), está fuertemente correlacionada con el desempeño de los precios de las acciones [...] Incluso entre las empresas con márgenes de beneficio similares, los accionistas de empresas que superan a sus pares en productividad de activos disfrutan de mayores rendimientos de acciones más altos que los asociados con los márgenes de ganancia” (p.33).

Según Parviz y Abolghasem (2012): "Uno de los criterios para evaluar la capacidad de gestión de una empresa para obtener retorno es la tasa de retorno de los activos (ROA), con respecto a los recursos existentes" (p.12).

Para Hunjra, Shahzad, Chani, Hassan y Mustafa (2014): "La volatilidad en los precios de las acciones es uno de los temas más discutidos en finanzas”, además agregan que: "Se han realizado muchos estudios para encontrar los factores que causan la fluctuación en los precios de las acciones y se han encontrado diferentes resultados" (p.109).

Lee y Zhang (2012), concluyen que:

En general, la evidencia sugiere que hay importantes recompensas tangibles para los accionistas de las empresas que logran mejoras de productividad en sus negocios. La evidencia debe brindar cierta comodidad a los gerentes y dueños de negocios que pueden ser escépticos acerca de si las mejoras de productividad realmente producen beneficios positivos para sus negocios más allá de los costos. (p.33)
Sin embargo, existen casos en el que no posee relación alguna, por lo que el inversionista debe realizar, adicionalmente, un análisis de los estados financieros y estado de resultados, para verificar la rentabilidad de la empresa o como lo expresa Kamar (2017): “[...] que los inversionistas en la implementación de la inversión no solo consideren el factor financiero, sino que también presten atención a otro como el político, el económico" (p.66).

Considerando lo que los autores señalan, surge el problema de analizar y comprobar si la rentabilidad financiera (ROE) y la rentabilidad operativa (ROA) son indicadores financieros que reflejan efectivamente el precio de la acción de una compañía, el cual es el indicador más fácil y directo de ser observado y cotejado por cualquier inversionista en la búsqueda de compra de acciones. Ante la situación descrita surge la interrogante planteando el siguiente problema de investigación:

¿Cómo influye la rentabilidad financiera y operativa cuando actúan de manera simultánea sobre el precio de la acción? Para responder la interrogante se plantean los siguientes objetivos:

Determinar el efecto simultáneo de la rentabilidad financiera y operativa sobre el precio de la acción de las empresas industriales como objetivo general, y como objetivos secundarios o específicos, determinar el efecto del ROE sobre el precio de la acción, y determinar el efecto del ROA sobre el precio de la acción de las empresas, de manera independiente cada ratio financiero.

Las hipótesis surgidas en la investigación son las siguientes:

Como hipótesis general:

Ho: La rentabilidad financiera y operativa simultáneamente no influyen positivamente sobre el precio de la acción de las empresas industriales en estudio.

Ha: La rentabilidad financiera y operativa simultáneamente influyen positivamente sobre el precio de la acción de las empresas industriales en estudio.

Las hipótesis específicas de la investigación son las siguientes: 1ra hipótesis específica

Ho: La rentabilidad financiera no influye positivamente en el precio de la acción

Ha: La rentabilidad financiera influye positivamente en el precio de la acción

2da hipótesis específica

Ho: La rentabilidad operativa no influye positivamente en el precio de la acción 
Ha: La rentabilidad operativa influye positivamente en el precio de la acción

La investigación se encuentra estructurada en tres secciones: materiales y métodos, resultados y discusión.

\section{MATERIAL Y MÉTODOS}

El diseño de estudio es no experimental, con enfoque cuantitativo. Es no experimental porque se recopiló información y los datos se procesaron directamente sin manipular experimentalmente las variables para obtener los resultados. Es cuantitativo debido a que los datos serán procesados estadísticamente para obtener los resultados de la investigación. El nivel de la investigación es transversal y de tipo correlacional. Es transversal porque abarcará la recopilación retrospectiva de datos en un periodo de 10 años desde el 2009 al 2018. Es correlacional porque se determinó la relación e influencia de la rentabilidad (financiera y operativa) en el precio de la acción. Las variables son las siguientes:

Variable independiente 1: Rentabilidad financiera

$$
\frac{\text { Beneficio neto }}{\text { Patrimonio }}
$$

Variable independiente 2: Rentabilidad operativa

$$
\frac{\text { Beneficio neto }}{\text { Total activo }}
$$

Variable Dependiente: Precio de la acción

\section{Precio x Acción}

La población de estudio son 34 empresas industriales peruanas que cotizan en la bolsa de valores de Lima. Desde los periodos 2009-2018. Las acciones y actividades que se realizaron para la ejecución del trabajo de investigación son las siguientes:

$1^{\circ}$ etapa: Recolección de datos

a. Se recolectó información financiera de las empresas, además del precio de sus acciones en el periodo 2009-2018

$2^{\circ}$ etapa: Localización

b. Se recolectaron los datos de la página web: https:// www.bvl.com.pe/

$3^{\circ}$ etapa: Modelos teóricos utilizados

c. Como modelos teóricos se utilizaron las investigaciones de:

- Pražák y Stavárek (2017)

- $\quad$ Milošević y Milenković (2017)
- $\quad \operatorname{Kamar}(2017)$

- Tamuntuan (2015)

- $\quad$ Barakat (2014)

- $\quad$ Hunjra et al. (2014)

- $\quad$ Lee y Zhang (2012)

- Parviz y Abolghasen (2012)

$4^{\circ}$ etapa: Análisis de datos

d. Se realizó el análisis de correlación para establecer como primer paso la existencia o no de relación entre las variables de estudio.

e. Posteriormente, se realizó el análisis de regresión simple y múltiple para observar el porcentaje de contribución de las variables ROE y ROA sobre el precio de la acción.

f. Se usó el análisis de la varianza (ANOVA) y la significancia de las variables para dar respuesta a las hipótesis planteadas.

g. Finalmente, se realizó el análisis de coeficientes para determinar los componentes de la recta.

$5^{\circ}$ etapa: Procesamiento de datos

h. La información recopilada fue tabulada en el software estadístico de Eviews.

La técnica es el análisis documental y el instrumento a utilizar es el análisis de contenido. Los análisis estadísticos que se llevaron a cabo son los siguientes:

- Análisis de correlación

- Análisis de regresión

- $\quad$ Análisis del ANOVA

- $\quad$ Análisis de coeficientes

\section{RESULTADOS}

Los resultados de la investigación inician con la presentación de la diversidad de rubros de empresas del sector industrial que se encuentra categorizado hasta la fecha en la Bolsa de Valores de Lima (2019). En la tabla 1, se muestran los tipos de empresas que formaron parte de la investigación:

Existe disparidad entre las empresas en relación a su actividad económica; se tienen empresas de alimentos, cementos, automotriz, petroleras, textiles y empresa editora 
Tabla 1

Rubros de las empresas del sector industrial de la Bolsa de Valores de Lima (BVL).

\begin{tabular}{lc}
\hline RUBROS & CANTIDAD \\
\hline Alimentos (extracción, producción, comercialización) & 9 \\
Cementos, aceros, cerámica, metales & 10 \\
Llantas, artículos de aseo, acumuladores y autopartes, bombas mecánicas & 8 \\
Envases & 1 \\
Petróleo, actividad minera & 3 \\
Textiles & 2 \\
Editora & 1 \\
TOTAL & 34 \\
\hline
\end{tabular}

Fuente: Elaboración propia.

Tabla 2

Interpretación del coeficiente de correlación.

\begin{tabular}{cl}
\hline $\begin{array}{c}\text { Valor del coeficiente } \\
\text { de correlación }\end{array}$ & \multicolumn{1}{c}{$\begin{array}{c}\text { Dirección y fuerza de la } \\
\text { correlación }\end{array}$} \\
\hline$-1,0$ & Perfectamente negativo \\
$-0,8$ & Fuertemente negativo \\
$-0,5$ & Moderadamente negativo \\
$-0,2$ & Débilmente negativo \\
0,0 & No existe asociación \\
$+0,2$ & Débilmente positivo \\
$+0,5$ & Moderadamente positivo \\
$+0,8$ & Fuertemente positivo \\
$+1,0$ & Perfectamente positivo \\
\hline
\end{tabular}

Fuente: (Zou, Tuncali y Silverman, 2003, p. 618).

como se observa en la Tabla 1. Se trabajó con una data histórica de las empresas en estudio de 10 años, abarcó desde el periodo 2009 al 2018 y fue obtenida del sitio web oficial de la Bolsa de Valores de Lima.

Para dar inicio a los resultados que darán respuesta a los objetivos e hipótesis planteadas se procedió como primer paso realizar el análisis de correlación, con la intencionalidad de verificar la existencia o no de relación entre las variables señaladas. Previamente para el análisis de correlación entre las variables, se presenta en la Tabla 2, los valores de los coeficientes de correlación con su respectivo significado de dirección y fuerza, que sirve de guía para la posterior interpretación de los resultados.

La escala de interpretación del coeficiente de correlación se observa en la Tabla 2, esta escala va desde - 1,0 (Perfectamente negativo) o que no existe correlación hasta $+1,0$ (Perfectamente positivo) o que existe una correlación intensa o fuerte. La escala servirá como referencia para determinar el grado de correlación que se presentan en las Tablas 3, 4 y 5, que poseen los resultados del análisis de correlación y regresión entre las variables de estudio (ROA, ROE y el precio de la acción (PA)).

En el análisis de correlación y regresión del ROE y el PA, se observa en la Tabla 3 que sí existe relación y el grado de correlación entre el ROE y el PA es igual a 0,2736 y de acuerdo a la interpretación de la Tabla 2 , indica que la dirección y fuerza de la correlación es Débilmente positiva, lo que significa que el ROE se relaciona con el PA, pero de una manera insignificante. En el análisis de regresión se observa que valor del $\mathrm{R}^{2}$ ajustado indica que el $\mathrm{ROE}$ explica en un 27,14\% a la variable precio de la acción. El valor de 0,000 en la Probabilidad (F-statistic) señala que su influencia es significativa para ser considerado el ROE en la ecuación de la recta del PA. Al parecer los resultados de la rentabilidad financiera no son determinantes para concluir que posee un impacto significativo sobre el precio de la acción. 
Tabla 3

Análisis del ROE sobre el precio de la acción.

\begin{tabular}{|c|c|c|c|}
\hline \multicolumn{4}{|c|}{ Dependent Variable: Precio de la acción (PA) } \\
\hline \multicolumn{4}{|c|}{ Method: Panel Least Squares } \\
\hline \multicolumn{4}{|l|}{ Sample: 20092018} \\
\hline \multicolumn{4}{|l|}{ Periods included: 10} \\
\hline \multicolumn{4}{|c|}{ Cross-sections included: 60} \\
\hline \multicolumn{4}{|c|}{ Total panel (unbalanced) observations: 340} \\
\hline R-squared & 0,273568 & Mean dependent var & 7,212962 \\
\hline Adjusted R-squared & 0,271419 & S.D. dependent var & 25,60418 \\
\hline S.E. of regression & 21,85495 & Akaike info criterion & 9,012597 \\
\hline Sum squared resid & 161441,9 & Schwarz criterion & 9,035120 \\
\hline Log likelihood & $-1530,141$ & Hannan-Quinn criter. & 9,021571 \\
\hline F-statistic & 127,2881 & Durbin-Watson stat & 0,118362 \\
\hline Prob(F-statistic) & 0,000000 & & \\
\hline
\end{tabular}

Fuente: Elaboración propia.

Tabla 4

Análisis del ROA sobre el precio de la acción.

\begin{tabular}{llll}
\hline $\begin{array}{l}\text { Dependent Variable: Precio de la acción (PA) } \\
\text { Method: Panel Least Squares }\end{array}$ & & \\
Sample: 20092018 & & & \\
Periods included: 10 & & & \\
Cross-sections included: 60 & & & \\
Total panel (unbalanced) observations: 340 & 0,262436 & Mean dependent var & 7,212962 \\
\hline R-squared & 0,260253 & S.D. dependent var & 25,60418 \\
Adjusted R-squared & 22,02178 & Akaike info criterion & 9,027806 \\
S.E. of regression & 163916,1 & Schwarz criterion & 9,050329 \\
Sum squared resid & $-1532,727$ & Hannan-Quinn criter. & 9,036780 \\
Log likelihood & 120,2651 & Durbin-Watson stat & 0,160524 \\
F-statistic & 0,000000 & & \\
Prob(F-statistic) & & & \\
\hline
\end{tabular}

Fuente: Elaboración propia

En el análisis del ROA sobre el PA, se observa en la Tabla 4, que el grado de correlación entre ambas variables es igual a 0,2624 , lo que indica que la dirección y fuerza de la correlación presenta una relación Débilmente positiva (Tabla 2). El valor de 0,000 en la Probabilidad (F-statistic) señala que la influencia del ROA en PA es significativa como para ser considerado el ROA en la ecuación de la recta del PA. En el análisis de regresión el valor del $\mathrm{R}^{2}$ ajustado indica que el ROA explica en un 26,03\% a la variable PA. De igual modo que con el ROE, el ROA no es determinante para concluir que la rentabilidad operativa posee un impacto significativo sobre el PA.
Analizando de manera simultánea el ROE y ROA sobre el precio de la acción, en la Tabla 5, se visualiza que sí existe relación entre el ROE y ROA con el precio de la acción (PA), su relación de 0,2791 refleja que es Débilmente positiva. El valor de 0,000 en la Probabilidad (F-statistic) señala que la influencia del ROE y ROA conjuntamente en el PA es significativa como para ser considerados en la ecuación de la recta del precio de la acción. En el análisis de regresión el valor del $\mathrm{R}^{2}$ ajustado indica que el ROE y ROA explican un 27,48 \% a la variable PA. Al explicar un valor menor al $50 \%$, señala que las variables ROE y ROA trabajando conjuntamente sobre el PA no poseen un im- 
Tabla 5

Análisis del ROE y ROA sobre el precio de la acción.

\begin{tabular}{|c|c|c|c|}
\hline \multicolumn{4}{|c|}{ Dependent Variable: Precio de la acción (PA) } \\
\hline \multicolumn{4}{|c|}{ Method: Panel Least Squares } \\
\hline \multicolumn{4}{|l|}{ Sample: 20092018} \\
\hline \multicolumn{4}{|l|}{ Periods included: 10} \\
\hline \multicolumn{4}{|c|}{ Cross-sections included: 60} \\
\hline \multicolumn{4}{|c|}{ Total panel (unbalanced) observations: 340} \\
\hline R-squared & 0,279113 & Mean dependent var & 7,212962 \\
\hline Adjusted R-squared & 0,274835 & S.D. dependent var & 25,60418 \\
\hline S.E. of regression & 21,80366 & Akaike info criterion & 9,010817 \\
\hline Sum squared resid & 160209,6 & Schwarz criterion & 9,044602 \\
\hline Log likelihood & $-1528,839$ & Hannan-Quinn criter. & 9,024279 \\
\hline F-statistic & 65,23994 & Durbin-Watson stat & 0,133926 \\
\hline Prob(F-statistic) & 0,000000 & & \\
\hline
\end{tabular}

Fuente: Elaboración propia.

Tabla 6

Análisis de coeficientes del ROE sobre el precio de la acción.

\begin{tabular}{|c|c|c|c|c|}
\hline \multicolumn{5}{|c|}{ Dependent Variable: Precio de la acción (PA) } \\
\hline \multicolumn{5}{|c|}{ Method: Panel Least Squares } \\
\hline \multicolumn{5}{|c|}{ Sample: 20092018} \\
\hline \multicolumn{5}{|c|}{ Periods included: 10} \\
\hline \multicolumn{5}{|c|}{ Cross-sections included: 60} \\
\hline \multicolumn{5}{|c|}{ Total panel (unbalanced) observations: 340} \\
\hline Variable & Coefficient & Std. Error & t-Statistic & Prob. \\
\hline C & $-1,815880$ & 1,430125 & $-1,269735$ & 0,2051 \\
\hline ROE & 82,96243 & 7,353390 & 11,28220 & 0,0000 \\
\hline
\end{tabular}

Fuente: Elaboración propia.

pacto significativo. Los resultados permitieron comprobar las hipótesis planteadas:

Comprobación de hipótesis general:

Ho: La rentabilidad financiera y operativa simultáneamente no influyen positivamente sobre el precio de la acción de las empresas industriales en estudio. SE RECHAZA

Ha: La rentabilidad financiera y operativa simultáneamente influyen significativamente sobre el precio de la acción de las empresas industriales en estudio. SE ACEPTA

Comprobación de hipótesis específicas:

1ra hipótesis específica

Ho: La rentabilidad financiera no influye significativamente en el precio de la acción. SE RECHAZA
Ha: La rentabilidad financiera influye positivamente en el precio de la acción. SE ACEPTA

2da hipótesis específica

Ho: La rentabilidad operativa no influye positivamente en el precio de la acción. SE RECHAZA

Ha: La rentabilidad operativa influye positivamente en el precio de la acción. SE ACEPTA

El análisis de coeficientes del ROE y ROA sobre el PA se presenta en las Tablas 6, 7 y 8.

En el análisis de coeficientes del ROE sobre el PA de la Tabla 6, el valor Beta de la constante del ROE es positivo por lo que señala que la influencia es positiva sobre el PA. El valor de significancia del ROE igual a 0,000 indica que es importante para ser considerado en la ecuación de la recta en comparación a la constante que indica lo contrario. 
Tabla 7

Análisis de coeficientes del ROA sobre el precio de la acción.

\begin{tabular}{|c|c|c|c|c|}
\hline \multicolumn{5}{|c|}{ Dependent Variable: Precio de la acción (PA) } \\
\hline \multicolumn{5}{|c|}{ Method: Panel Least Squares } \\
\hline \multicolumn{5}{|c|}{ Sample: 20092018} \\
\hline \multicolumn{5}{|c|}{ Periods included: 10} \\
\hline \multicolumn{5}{|c|}{ Cross-sections included: 60} \\
\hline \multicolumn{5}{|c|}{ Total panel (unbalanced) observations: 340} \\
\hline Variable & Coefficient & Std. Error & t-Statistic & Prob. \\
\hline C & $-3,964139$ & 1,570069 & $-2,524818$ & 0,0120 \\
\hline ROA & 189,5496 & 17,28435 & 10,96654 & 0,0000 \\
\hline
\end{tabular}

Fuente: Elaboración propia.

Tabla 8

Análisis de coeficientes del ROE y ROA sobre el precio de la acción.

Dependent Variable: Precio de la acción (PA)

Method: Panel Least Squares

Sample: 20092018

Periods included: 10

Cross-sections included: 60

Total panel (unbalanced) observations: 340

\begin{tabular}{lcccc}
\hline Variable & Coefficient & Std. Error & t-Statistic & Prob. \\
C & $-2,964558$ & 1,595206 & $-1,858417$ & 0,0640 \\
ROE & 54,09400 & 19,37311 & 2,792222 & 0,0055 \\
ROA & 72,76054 & 45,19207 & 1,610029 & 0,1083 \\
\hline
\end{tabular}

Fuente: Elaboración propia.

Tabla 9

Ecuación de la recta de las variables de estudio.

\begin{tabular}{lll}
\hline Variables & Tipo de regresión & Ecuación de la recta \\
\hline ROE en PA & Simple & PA $=82,9624$ ROE \\
ROA en PA & Simple & PA $=-3,9641+189,5496$ ROA \\
ROE y ROA en PA & Múltiple & PA $=54,09400$ ROE $+72,76054$ ROA \\
\hline
\end{tabular}

Fuente: Elaboración propia.

El análisis de coeficientes del ROA sobre el PA de la Tabla 7, se muestra el valor Beta de la constante del ROA es positivo por lo que señala que la influencia es positiva sobre el PA. Los valores de significancia del ROA y de la constante son menores a 0,05 señalando que ambos son importantes para ser considerados en la ecuación de la recta. En la Tabla 8, se muestra el análisis de coeficientes del ROE y ROA sobre el PA.

El análisis de coeficientes del ROE, ROA sobre el PA en la Tabla 8, muestra que el valor Beta de las variables ROE y
ROA, son positivos indicando que su influencia es positiva sobre el PA. El valor de significancia del ROE, indica que es importante para ser considerado en la ecuación de la recta, a diferencia del ROA y de la constante cuyos valores superan el mínimo de 0,05. En la Tabla 9, se procedió a generar las ecuaciones de las rectas.

Las ecuaciones de la recta mostradas en la Tabla 9, fueron obtenidas a partir de los datos tabulados en el análisis de coeficientes de las variables. 


\section{DISCUSIÓN}

Los resultados de las variables ROE y ROA tuvieron una influencia positiva sobre el PA, pero solo lo explican en un $27,14 \%$ y $26,03 \%$, independientemente y un $21,80 \%$ de manera simultánea (ROE y ROA), señalando que la contribución es pequeña o insignificante. El resultado coincide con las investigaciones de Hunjra et. al. (2014), Milošević Avdalović y Milenković (2017) y Tamuntuan (2015), que concluyeron que el ROE y el ROA, muestran un impacto positivo pero una contribución insignificante o moderada en el precio de la acción. Sin embargo, los autores Kamar (2017), Barakat (2014), Pražák y Stavárek (2017) y Parviz y Abolghasen (2012), no concuerdan con los resultados debido a que demostraron que el ROE y ROA, sí tienen una relación positiva y además una contribución significativa en el PA. Una de las razones del bajo grado de influencia lo señalan Satryo, Rokhmania y Diptyana (2016), los inversores tienen más en cuenta los ratios del mercado, que los ratios de rentabilidad y de solidez en la toma de decisiones de inversión en el mercado. Entre las causas se tiene la actividad principal de la empresa; en la investigación se han considerado las empresas del sector industrial y en este sector existen empresas que pertenecen a distintos rubros como el textil, alimentos, químicos, cementos, petroleras entre otros, y por razones económicas y/o actividades de operación el valor de las variables de estudio (ROA, ROE y PA) es diverso y particular para cada empresa, creando mucha disparidad, que además no ejercen influencia significativa en el precio de las acciones .

\section{REFERENCIAS BIBLIOGRÁFICAS}

Barakat, A. (2014). The Impact of Financial Structure, Financial Leverage and Profitability on Industrial Companies Shares Value (Applied Study on a Sample of Saudi Industrial Companies). Research Journal of Finance and Accounting, 5(1), 55-66.

Bolsa de Valores de Lima. (2019). Empresas con valores listados.Sector industrial.Lima. Obtenido de https:// www.bvl.com.pe/mercempresasporsector.html\#

Hunjra, A.., Shahzad, M., Chani, M., Hassan, S., \& Mustafa, U. (2014). Impact of Dividend Policy, Earning per
Share, Return on Equity, Profit after Tax on Stock prices. International Journal of Economics and Empirical Research, 2(3), 109-115.

Kamar, K. (2017). Analysis of the Effect of Return on Equity (ROE) and Debt to Equity Ratio (DER) On Stock Price on Cement Industry Listed In Indonesia Stock Exchange (Idx) In the Year of 2011-2015. IOSR Journal of Business and Management (IOSR-JBM), 19(5), 6676. doi:10.9790/487X-1905036676

Lee, A., \& Zhang, T. (2012). Productivity, Return-on-capital and Stock Price. In Accountig and Productivity. (T. Suwardy, \& G. Pan, Eds.) Singapore: Singapore Management University.

Milošević, S., Avdalović, A. \& Milenković, I. (2017). Impact of Company Performances on the Stock Price: An Empirical Analysis on select companies in Serbia. Economics of Agriculture, 64(2), 561-570.

Parviz, S., \& Abolghasem, O. (2012). Studying the effect of assets return rate on stock price of the companies accepted in Tehran stock exchange. BEH - Business and Economic Horizons, 8(2), 12-22. doi:http://dx.doi. org/10.15208/beh.2012.7

Pražák, T., \& Stavárek, D. (2017). The effect of financial ratios on the stock price development. Katowise: Silesian University.

Satryo, A., Rokhmania, N., \& Diptyana, P. (2016). The influence of profitability ratio, market ratio, and solvency ratio on the share prices of companies listed on LQ 45 Index. The Indonesian Accounting Review, 6(1), 55-66.

Tamuntuan, U. (2015). Analysing the effect of return on equity, return on assets and earnings per share toward share price: an emperical study of food and beverage companies listed on indonesia stock exchange. Journal Berkala Ilmiah Efisiensi, 15(5), 446-457.

Zou, K., Tuncali, K., \& Silverman, S. (2003). Correlation and simple linear regression. Radiology. 227(3), 617-628. 